\title{
Transport of inertial particles by Lagrangian coherent structures: application to predator-prey interaction in jellyfish feeding
}

\author{
J. PEN G ${ }^{1} \dagger$ AND J. O. DABIR I I ${ }^{1,2}$ \\ ${ }^{1}$ Bioengineering, California Institute of Technology, Pasadena, CA 91125, USA \\ ${ }^{2}$ Graduate Aeronautical Laboratories, California Institute of Technology, Pasadena, \\ CA 91125, USA
}

(Received 29 May 2008 and in revised form 25 September 2008)

We use a dynamical systems approach to identify coherent structures from often chaotic motions of inertial particles in open flows. We show that particle Lagrangian coherent structures (pLCS) act as boundaries between regions in which particles have different kinematics. They provide direct geometric information about the motion of ensembles of inertial particles, which is helpful to understand their transport. As an application, we apply the methodology to a planktonic predator-prey system in which moon jellyfish Aurelia aurita uses its body motion to generate a flow that transports small plankton such as copepods to its vicinity for feeding. With the flow field generated by the jellyfish measured experimentally and the dynamics of plankton described by a modified Maxey-Riley equation, we use the pLCS to identify a capture region in which prey can be captured by the jellyfish. The properties of the pLCS and the capture region enable analysis of the effect of several physiological and mechanical parameters on the predator-prey interaction, such as prey size, escape force, predator perception, etc. The methods developed here are equally applicable to multiphase and granular flows, and can be generalized to any other particle equation of motion, e.g. equations governing the motion of reacting particles or charged particles.

\section{Introduction}

The advection of finite-size or inertial particles in open, unsteady flow is often chaotic (e.g. Aref 1984; Wiggins 1992). Compared with ideal (infinitesimal) fluid tracers that simply follow local flow velocities, the inertia of finite-size particles makes their motion lag fluid motion. There are additional forces acing on finite-size particles such as buoyancy, Stokes drag, the added mass, and the Basset-Boussinesq memory force. In a classic paper, Maxey \& Riley (1983) summarized these forces and introduced the equation of motion for small rigid spherical particles in an incompressible fluid. A widely used reduced form of the Maxey-Riley equation (with Basset-Boussinesq memory force and $\nabla^{2} u$ terms neglected) is

$$
\frac{\mathrm{d} \boldsymbol{v}}{\mathrm{d} t}-\frac{3 R}{2} \frac{\mathrm{D} \boldsymbol{u}}{\mathrm{D} t}=-A(\boldsymbol{v}-\boldsymbol{u})+\left(1-\frac{3 R}{2}\right) \boldsymbol{g}
$$


with

$$
R=\frac{2 \rho_{f}}{\rho_{f}+2 \rho_{p}}, \quad A=\frac{R}{S t}, \quad S t=\frac{2}{9}\left(\frac{a}{L}\right)^{2} R e .
$$

Equation (1.1) is made dimensionless with characteristic length scale $L$, characteristic velocity $U$, and characteristic time scale $T=L / U$. The variable $v$ represents the velocity of the particle, $\boldsymbol{u}$ that of the fluid, $\rho_{p}$ the density of the particle, $\rho_{f}$ the density of the fluid, $v$ the kinematic viscosity of the fluid, $a$ the radius of the particle, and $g$ gravity. The particle dynamics depend on three dimensionless parameters: the mass ratio parameter $R$, the particle Stokes number $S t$, and the Reynolds number $R e=U L / \nu$. The size parameter $A$ describes the effect of particle inertia, with the limit $A \rightarrow \infty$ corresponding to infinitesimal fluid tracers $(a=0)$. The derivative $\mathrm{D} \boldsymbol{u} / \mathrm{D} t$ is taken along the path of an ideal fluid tracer

$$
\frac{\mathrm{D} \boldsymbol{u}}{\mathrm{D} t}=\frac{\partial \boldsymbol{u}}{\partial t}+(\boldsymbol{u} \cdot \nabla) \boldsymbol{u},
$$

whereas the derivative $\mathrm{d} \boldsymbol{v} / \mathrm{d} t$ is taken along the trajectory of the particle. A detailed description and discussion of the Maxey-Riley equation can be found in the review by Michaelides (1997).

The Maxey-Riley equation provides a theoretical tool to study dynamical systems of inertial particles in fluid flows. Dynamical systems of small inertial particles described by the Maxey-Riley equation are higher dimensional $(2 d+1)$ than those of ideal fluid tracers $(d+1)(d$ is the dimensionality of the flow, see Benczik, Toroczkai \& Tel 2003). The motion of an inertial particle in the fluid often differs from that of an ideal tracer. In fact the Lagrangian trajectories between the two can differ drastically. The difference not only depends on properties of particles, but also on the characteristics of flow fields. For example, the distinction between inertial particle trajectories and fluid trajectories is most significant in those regions where the flow has hyperbolic stagnation points (Babiano et al. 2000).

Some interesting features of dynamical systems of inertial particles include clustering and dispersion of particles. For small Stokes number, there exists an invariant manifold to which inertial particles are asymptotically attracted. They are called attracting slow manifolds (Haller \& Sapsis 2008), to distinguish them from classic fluid manifolds that attract ideal fluid tracers (Rom-Kedar, Leonard \& Wiggins 1990). The attracting manifolds are very useful in finding particle clustering locations in open flows. However, knowledge of where the particles come from is equally important as knowing where the particles go. In other words, it is of interest to derive a topological map that separates particles into different groups, depending on their later kinematics.

To achieve this, we use the finite-time Lyapunov exponent (FTLE) to identify geometric separatrices from trajectories of inertial particles. We define these separatrices, which are called particle Lagrangian coherent structures (pLCS), and show that pLCS formulate a unique geometry indicating transport of inertial particles in open flows. The new method enables analysis of transport of inertial particles, thereby extending previous studies in which FTLE and fluid Lagrangian coherent structures (LCS) were used to understand fluid transport in chaotic flows (Haller 2002; Shadden, Lekien \& Marsden 2005; Shadden, Dabiri \& Marsden 2006; Franco et al. 2007).

As a demonstration, we apply the new method to a marine predator-prey system. In this system, the predator, moon jellyfish Aurelia aurita, feed on small planktonic prey such as copepods. The system is unique in that jellyfish use body movements to 
generate flow currents, which induce transport of prey towards the tentacles and oral arms, where the prey can be captured. We identify a capture region using the concept of pLCS. Prey inside the region are captured by the jellyfish while prey outside the region are able to escape. The properties of pLCS also enable the analysis of the effects of prey size and escape force on the size of capture region.

The paper is organized as follows: $\S 2$ presents an equation of motion modified from the Maxey-Riley equation to account for the self-propulsion of prey. This is followed by a presentation of the calculation of FTLE and pLCS from inertial particle trajectories. Section 3 reports results obtained from the case study of jellyfish predation. The effects of prey size and escape forces on the capture region are investigated by comparison with the capture region based on ideal fluid tracers. The paper concludes with a discussion of the benefits and limitations of the developed methods and suggestions for potential applications in $\S 4$.

\section{Methods}

\subsection{Flow generation and measurement}

In the present work, we study the predation of moon jellyfish Aurelia aurita. These animals are cruising predators that feed on small plankton. They use periodic contraction and relaxation of their bells to swim. As the animal moves forward, the flow induced by body motion also facilitates the transport of prey toward capture surfaces under the bell. Using digital particle image velocimetry (DPIV), we measured the flow field generated by five free-swimming Aurelia aurita in a 75 gallon water tank. Details of the experimental methods were similar to recent studies involving the same species of animal (Shadden et al. 2006; Franco et al. 2007). DPIV measurements were collected on the symmetry plane of the animal for several consecutive swimming cycles.

\subsection{Dynamics of prey}

Aurelia aurita feed on microcrustaceans, such as copepods. These animals have a characteristic length of $a=1 \mathrm{~mm}$. For simplicity, we neglected their body shape and considered them as spherical particles. Hence their dynamics could be described by the Maxey-Riley equation. However, the forces on the right-hand side of the Maxey-Riley equation only include fluid forces acting on passive particles due to the background fluid currents and gravity. Small plankton are capable of self-propulsion, often triggered by perception of a nearby predator. This effect was incorporated in this study by the addition of a term $\boldsymbol{a}_{e}=\boldsymbol{F}_{e} / m$ into (1.1), representing the acceleration rate of prey animals due to the self-generated escape force $\boldsymbol{F}_{e}$, with $m$ being the mass of individual prey. This escape force may be dependent on time and the local flow characteristics, e.g. local shear stress. Because most microscopic aquatic animals are neutrally buoyant, we took the mass ratio parameter $R$ in (1.1) equal to $2 / 3$. Therefore the dynamics of a prey animal can be expressed as

$$
\frac{\mathrm{d} \boldsymbol{v}}{\mathrm{d} t}-\frac{\mathrm{D} \boldsymbol{u}}{\mathrm{D} t}=-A(\boldsymbol{v}-\boldsymbol{u})+\boldsymbol{a}_{e} .
$$

Without the escape acceleration term $\boldsymbol{a}_{e},(2.1)$ is the linearized Maxey-Riley equation for neutrally buoyant spherical particles. The equation was non-dimensionalized by length scale $L=5 \mathrm{~cm}$ (the radius of the jellyfish) and characteristic velocity $U=1 \mathrm{~cm} \mathrm{~s}^{-1}$ (the jellyfish swimming speed). The corresponding Reynolds number 
of the flow $R e=500$, and for a prey with size $a=1 \mathrm{~mm}$, the particle Stokes number $S t=0.04$ and the particle size parameter $A=15$.

\subsection{Transport of prey}

Similar to the method for locating fluid LCS, the FTLE field was calculated from inertial particle trajectories to determine the pLCS. The FTLE field measures particle separation rate in the flow. The ridges on FTLE contour plots, indicating local maxima of separation rate, identify the pLCS.

For a given flow field $\boldsymbol{u}(t, \boldsymbol{x})$, the velocity of an inertial particle $\boldsymbol{v}\left(t ; t_{0}, \boldsymbol{x}_{0}\right)$ can be solved along its trajectory. The notation $\boldsymbol{v}\left(t ; t_{0}, \boldsymbol{x}_{0}\right)$ denotes the velocity at time $t$ of a particle starting at point $\boldsymbol{x}_{0}$ at time $t_{0}$. Note that the velocity of the flow $\boldsymbol{u}$ is Eulerian, whereas the velocity of the particle $v$ is Lagrangian. The trajectory of the inertial particle $\boldsymbol{x}\left(t ; t_{0}, \boldsymbol{x}_{0}\right)$ is the solution of

$$
\dot{\boldsymbol{x}}\left(t ; t_{0}, \boldsymbol{x}_{0}\right)=\boldsymbol{v}\left(t ; t_{0}, \boldsymbol{x}_{0}\right)
$$

with initial conditions

$$
\boldsymbol{x}\left(t_{0} ; t_{0}, \boldsymbol{x}_{0}\right)=\boldsymbol{x}_{0}, \quad \dot{\boldsymbol{x}}\left(t_{0} ; t_{0}, \boldsymbol{x}_{0}\right)=\boldsymbol{v}_{0},
$$

where the overdot denotes a time derivative. The initial velocity of a prey particle $\boldsymbol{v}_{0}$ was assumed to be equal to the local flow velocity. Together with (2.1), the trajectory can be calculated by integrating its velocity $\boldsymbol{v}\left(t ; t_{0}, \boldsymbol{x}_{0}\right)$ over time. A fourth order Runge-Kutta integration was used to calculate trajectories.

To avoid confusion between particle trajectories and spatial coordinates, the notation $\phi$ is used for particle trajectories and $\boldsymbol{x}$ for spatial coordinates. By following particle trajectories over a duration of time $T$ after initial time $t$, we obtain a particle flow map $\phi_{t}^{t+T}(\boldsymbol{x})$ that takes particles from their position $\boldsymbol{x}$ at initial time $t$ to their position at time $(t+T)$.

To locate the pLCS, we compute the FTLE field of the particle flow map. Consider the trajectories for a slightly perturbed particle at $\boldsymbol{y}=\boldsymbol{x}+\delta \boldsymbol{x}(0)$ at time $t$. After a time interval $T$, this perturbation becomes

$$
\delta \boldsymbol{x}(T)=\phi_{t}^{t+T}(\boldsymbol{y})-\phi_{t}^{t+T}(\boldsymbol{x})=\frac{\mathrm{d} \phi_{t}^{t+T}(\boldsymbol{x})}{\mathrm{d} \boldsymbol{x}} \delta \boldsymbol{x}(0)+O\left(\|\delta \boldsymbol{x}(0)\|^{2}\right),
$$

where $\left(\mathrm{d} \phi_{t}^{t+T}(\boldsymbol{x})\right) / \mathrm{d} \boldsymbol{x}$ is the deformation gradient tensor $\boldsymbol{D}$, calculated as $D_{i j}=\mathrm{d} \phi_{i} / \mathrm{d} x_{j}$. By dropping higher-order terms of $\delta \boldsymbol{x}(0)$, the magnitude of the perturbation is given by

$$
\|\delta \boldsymbol{x}(T)\|=\sqrt{\delta \boldsymbol{x}(0) \cdot \boldsymbol{\Delta} \cdot \delta \boldsymbol{x}(0)}
$$

where

$$
\boldsymbol{\Delta}=\frac{\mathrm{d} \phi_{t}^{t+T}(\boldsymbol{x})^{*}}{\mathrm{~d} \boldsymbol{x}} \cdot \frac{\mathrm{d} \phi_{t}^{t+T}(\boldsymbol{x})}{\mathrm{d} \boldsymbol{x}}
$$

is the Cauchy-Green deformation tensor. The operator '.' indicates the inner vector product and the superscript * denotes the transpose of a tensor. The magnitude of the perturbation is maximum when $\delta \boldsymbol{x}(0)$ is aligned with the eigenvector associated with the maximum eigenvalue of $\boldsymbol{\Delta}$. That is, if $\lambda_{\max }(\boldsymbol{\Delta})$ is the square root of the maximum eigenvalue of $\boldsymbol{\Delta}$, then

$$
\|\delta \boldsymbol{x}(T)\|=\lambda_{\max }(\boldsymbol{\Delta})\|\delta \boldsymbol{x}(0)\| .
$$


(a)

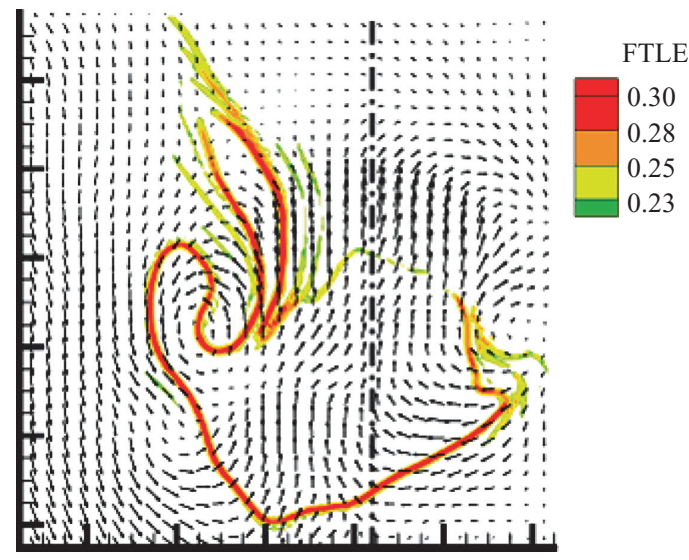

(b)

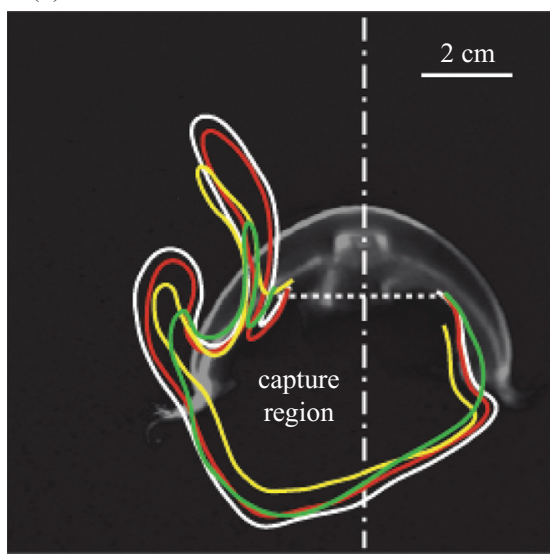

FIGURE 1. (a) The FTLE field (contour plot) and velocity field (vector plot) at a given time instant. (b) pLCS for different prey, superimposed over the jellyfish. Solid white: pLCS for ideal infinitesimal prey; red: pLCS for prey with size $a=1 \mathrm{~mm}$ and without escape force; yellow: pLCS for prey $(a=1 \mathrm{~mm})$ with an escape acceleration $\left(\boldsymbol{a}_{e}=0.05 \mathrm{~m} \mathrm{~s}^{-2}\right)$ opposite to local flow velocity; green: $\mathrm{pLCS}$ for prey $(a=1 \mathrm{~mm})$ with an escape acceleration $\left(\boldsymbol{a}_{e}=0.05 \mathrm{~m} / \mathrm{s}^{-2}\right)$ normal to local flow velocity. The capture regions are regions inside pLCS, closed by the imaginary dashed line on the top. The axis of the jellyfish body is shown as the dash-dot line.

The FTLE, representing the maximum linear growth rate of a small perturbation, is defined as

$$
\sigma_{t}^{t+T}(\boldsymbol{x})=\frac{1}{T} \ln \left\|\frac{\delta \boldsymbol{x}(T)}{\delta \boldsymbol{x}(0)}\right\|=\frac{1}{T} \ln \lambda_{\max }(\boldsymbol{\Delta}) .
$$

The LCS can be defined as a ridge line of the FTLE. Intuitively, a ridge line is a curve normal to which the topography is a local maximum. This is readily identified from the FTLE contour plot. For a precise definition of a ridge line, refer to Shadden et al. (2005).

In (2.8), the FTLE $\sigma(\boldsymbol{x})$ is not explicitly written as a function of the integration time $T$ because the length of integration time does not affect the location of the $\mathrm{pLCS}$. However, longer integration time can help determine the pLCS locations more accurately by better resolving the ridges of local maxima in the FTLE contour plot. The appropriate length of integration time depends on the particular flow being analysed, but in practice, the integration time in any pLCS analysis should be chosen such that the pLCS is clearly identifiable on the FTLE contour plot. The magnitude of the integration time $T$ in the present study is approximately 4 swimming cycles.

\section{Results}

First, we consider ideal infinitesimal $(a=0)$ prey with no mass. They are identical to ideal fluid tracers. The FTLE field is calculated based on the DPIV measurement of the flow generated by the jellyfish. Figure 1 $(a)$ shows the FTLE field for a jellyfish at a given time instant. The ridge of the FTLE contour plot is clearly identifiable as the pLCS, plotted as the solid white line in figure $1(b)$ around the jellyfish. The pLCS is a continuous curve that encloses a subumbrellar region in contact with the sensory apparatus of the animal and finger-like structure above the bell called 'lobes' (Shadden et al. 2006). The pLCS is not closed at the top as new lobes are 
continuously being generated. Even with the opening at the top, it is not conceptually difficult to separate the regions inside and outside the pLCS. The region inside the pLCS is the capture region, and ideal infinitesimal prey can be sampled and captured by the animal. Notice that the pLCS is not symmetric on figure 1(b). The most significant difference is that lobes are not well resolved on the right-hand side of the jellyfish body axis (the dash-dot line the figure 1). This is an artifact of the DPIV measurements, in which laser illumination was weaker on the right-hand side of the animal body (i.e. the laser sheet was introduced from the left). In the following, we only use the pLCS on the left-hand side of the jellyfish body axis to calculate the size of the capture region.

The pLCS for ideal infinitesimal prey is used as the baseline and the effect of prey inertia and escape forces on jellyfish feeding can be studied by comparisons among the pLCS. First, we study the effect of prey size/inertia on the capture region. The escape force by prey is not considered here, so $\boldsymbol{a}_{e}=0$ in (2.1). We consider prey size of $a=1 \mathrm{~mm}$, which is the characteristic size of copepods (Strickler 1975). The corresponding FTLE was calculated and pLCS was identified using the method described in $\S 2$. The pLCS and capture region are shown in figure 1(b).

We next consider the effects of prey escape forces on the capture region. Some microplankton, like copepods, can generate momentary acceleration up to $12 \mathrm{~m} \mathrm{~s}^{-2}$ when escaping predation by power-stroke of their legs (Strickler 1975). To successfully escape predation, the prey escape reaction should be directional. However, there is no existing literature demonstrating the directional preference of plankton's escape reaction to a predator, if any. To simplify the problem, we assume the escape force has one of the two different forms: $\boldsymbol{a}_{e}=-a_{e} \boldsymbol{u} / u$ and $\boldsymbol{a}_{e}=-a_{e} \boldsymbol{n} \times \boldsymbol{u} / u$ ( $\boldsymbol{n}$ being a unit vector normal to the plane). The former represents an escape force with its direction always opposite to local flow velocity, and the latter one with its direction always normal to local flow velocity and away from the predator. It is also assumed that the escape force is persistent, and thus the acceleration has a smaller value, with the magnitude $a_{e}=0.05 \mathrm{~m} \mathrm{~s}^{-2}\left(a_{e}=25\right.$ if non-dimensionalized by $\left.U^{2} / L\right)$ in both models. The pLCS are shown in figure 1(b): the yellow curve is the pLCS for prey with escape force opposite to local flow velocity, and the green curve is the pLCS for prey with escape force normal to local flow velocity.

Comparing the results plotted in figure $1(b)$, it is clear that the capture region for finite-sized prey is smaller than that for ideal infinitesimal prey. Adding an escape force to the prey can further reduce the area of the capture region. This is also true for four other animals studied. Comparisons between capture regions for ideal infinitesimal prey and for prey $(a=1 \mathrm{~mm})$ with an escape acceleration $\left(\boldsymbol{a}_{e}=0.05 \mathrm{~m} \mathrm{~s}^{-2}\right)$ opposite to local flow velocity are plotted for these animals in figure 2 . In table 1 , the area and the volume of the capture region are compared to study the effects of prey inertial and escape force. The area of the capture region is the area inside the pLCS on the left-hand side of the jellyfish body axis and the volume of the capture region is calculated based on the left-hand side pLCS assuming that pLCS is axisymmetric. For each individual animal, the sizes of the capture region area/volume are nondimensionalized by that for ideal infinitesimal prey. The average and the standard deviation of the five animals studied are listed in table 1. The size of the capture region indicates how effectively prey are captured. Thus it is more difficult for a jellyfish to capture finite-size prey than ideal, infinitesimal prey. The capability to generate a directional escape force further enhances prey escape from the predator, consistent with physical intuition.

The results shown above assumed that the escape response starts at the beginning of calculation, i.e. the prey constantly exhibits the escape response. However, in 
Prey characteristics

$a=0, a_{e}=0$

$a=1 \mathrm{~mm}, \boldsymbol{a}_{e}=0$

$a=1 \mathrm{~mm}, \boldsymbol{a}_{e}=0.05 \mathrm{~m} \mathrm{~s}^{-2}(\mathrm{O})$

$a=1 \mathrm{~mm}, \boldsymbol{a}_{e}=0.05 \mathrm{~m} \mathrm{~s}^{-2}(\mathrm{~N})$
Area of capture region Volume of capture region

1

$0.93 \pm 0.04$

$0.74+0.04$

$0.78 \pm 0.05$
1

$0.90 \pm 0.05$

$0.64 \pm 0.06$

$0.68 \pm 0.07$

TABLE 1. Areas of capture regions for different types of prey, normalized to that of ideal infinitesimal prey. $(\mathrm{O})$ represents $\boldsymbol{a}_{e}$ opposite to the flow direction and $(\mathrm{N})$ represents $\boldsymbol{a}_{e}$ normal to the flow direction. The numbers are shown in the form of the average and the standard deviation from the five animals studied.
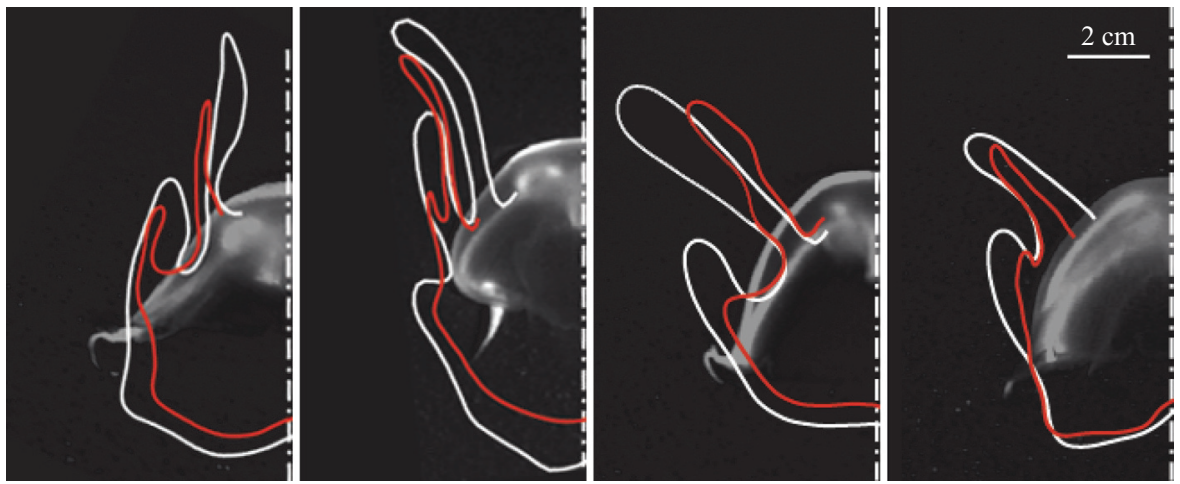

FIGURE 2. Capture regions for four additional jellyfish individuals of the same species. White: pLCS for ideal infinitesimal prey; red: pLCS for prey $(a=1 \mathrm{~mm})$ with escape acceleration $\left(\boldsymbol{a}_{e}=0.05 \mathrm{~m} \mathrm{~s}^{-2}\right)$ opposite to local flow velocity. Animals assume arbitrary swimming directions, but images are rotated so that the body axes are vertical. Only the half-plane on one side of the body axis (the dash-dot line) is shown. All images are of the same scale.

reality this is not the case. Studies show that escape reaction is usually inhibited in the absence of a predator because it requires up to 400 times the normal energetic expenditure for copepods (Strickler 1975; Fields \& Yen 1997). Thus escape reaction is elicited upon the perception of a measurable fluid mechanical signal in the flow generated by the predator. The signal can be disturbance in velocity or acceleration, but the most established signal is flow shear rate. A threshold value for shear rate, 1.5 to $8 \mathrm{~s}^{-1}$, is required to initiate escape reaction for copepods (Fields \& Yen 1997). The contour plot of shear rate of the flow generated by the jellyfish in figure 1 is shown in figure $3(a)$. The regions with high shear are in the vicinity of the jellyfish, which implies that prey might not be able to perceive the disturbance until they are close to the predator. To study the effect of the threshold value on the escape from predator, we calculated the pLCS and capture regions for different thresholds for shear perception $\tau_{t h}=0,1 \mathrm{~s}^{-1}$, and $\infty$. Threshold value for shear $\tau_{t h}=0$ is equivalent to a consistent escape response, while $\tau_{t h}=\infty$ is equivalent to no escape response. The prey has a size of $a=1 \mathrm{~mm}$ with an escape acceleration $\boldsymbol{a}_{e}=0.05 \mathrm{~m} \mathrm{~s}^{-2}$ and opposite to local flow velocity. It is assumed that prey do not initiate escape response until they perceive the local shear rate higher than the threshold. After the escape response is initiated, it persists even if the local shear rate drops below the threshold. The pLCS and capture regions are plotted in figure $3(b)$ : the white curve is the pLCS for prey with perception threshold $\tau_{t h}=0$; the red curve is the pLCS 
Prey characteristics

$\tau_{t h}=\infty$ (no response)

$\tau_{t h}=1 \mathrm{~s}^{-1}$

$\tau_{t h}=0$
Area of capture region

1

$0.94 \pm 0.02$

$0.81 \pm 0.04$
Volume of capture region

1

$0.91 \pm 0.03$

$0.72 \pm 0.06$

TABLE 2. Areas of capture regions for prey with different perception threshold values, normalized with the case of $\tau_{t h}=\infty$ (no response). The prey has a size of $a=1 \mathrm{~mm}$ with an escape acceleration $\boldsymbol{a}_{e}=0.5 \mathrm{~m} \mathrm{~s}^{-2}$ opposite to local flow velocity. The numbers are shown in the form of the average and the standard deviation from the five animals studied.

(a)

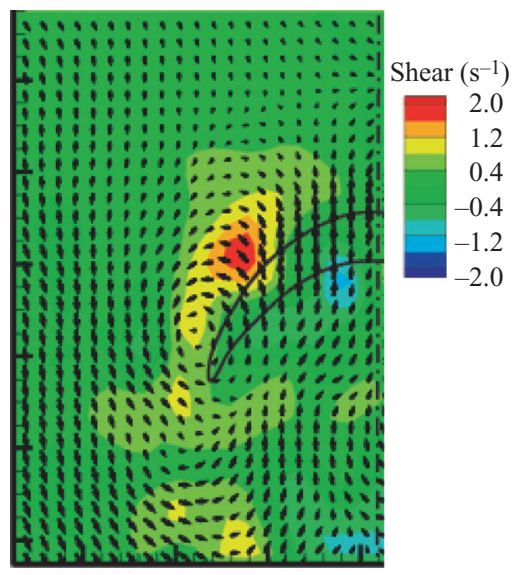

(b)

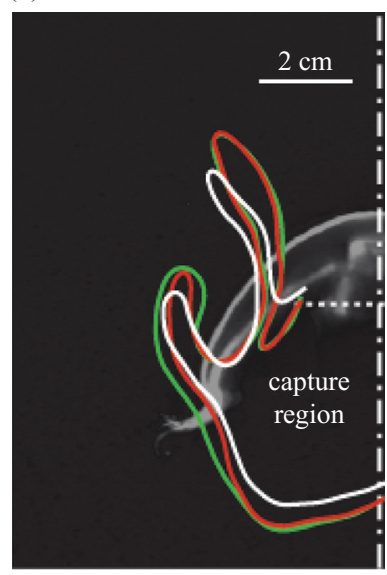

FIgURE 3. (a) Shear rate of the flow. The position of the jellyfish is given by its surface. $(b)$ pLCS and capture regions for prey with different perception threshold values. White: $\tau_{t h}=0$ (constant escape response); red: $\tau_{t h}=1 \mathrm{~s}^{-1}$; green: $\tau_{t h}=\infty$ (no escape response). Only the half-plane on one side of the body axis (the dash-dot line) is shown.

for prey with $\tau_{t h}=1 \mathrm{~s}^{-1}$; and the green curve is the pLCS for prey with $\tau_{t h}=\infty$. The sizes of capture regions for prey with different perception threshold are listed in table 2, normalized with the case of $\tau_{t h}=\infty$ (no escape response) for the individual animals. As the threshold value for perception decreases, the size of capture region also decreases. This is because prey are able to initiate the escape response earlier if they can perceive the shear rate at a lower level, thus enabling escape from the predator.

\section{Discussion}

In this study, a modified Maxey-Riley equation is used to describe the motion of inertial prey particles in the flow generated by a predator jellyfish. The equation applies to solid spherical particles with size much smaller than the characteristic length scale of the flow. For small particles, the effect of the flow field due to particle motion is restricted to a small area around the particle. This enables us to consider the flow field as independent of particle motion. In the case study of jellyfish feeding on copepods, we are able to measure the flow generated by the jellyfish and then subsequently calculate the motion of prey particles.

With the dynamics of prey particles specified, the study proposes a new method to study the fluid mechanical basis of jellyfish feeding on planktonic organisms. FTLE 
and pLCS are used to identify a capture region in the flow. By modelling different prey particles, we found that compared with ideal infinitesimal prey, the capture regions for prey with inertia and escape force can be significantly smaller. The differences (up to $26 \%$ or more, table 1$)$ are outside the calculation uncertainty $(3 \%)$, of which the major part is introduced when extracting pLCS from the FTLE plot (figure 1).

Previous models of jellyfish feeding appeal to a marginal flow mechanism, which relies on the comparison between prey escape speeds and flow velocities at jellyfish bell margins (Costello \& Colin 1994; Sullivan, Garcia \& Klein-MacPhee 1994). Prey with slow escape speeds are drawn to capture surfaces by flow generated by jellyfish at their bell margins. In contrast, the present model directly incorporates prey size, escape forces and perception threshold. The results show that due to inertia, some prey that would be captured if infinitesimal are not captured by the jellyfish, mostly those near the boundary of the capture region. This result is consistent with physical intuition, and it demonstrates that the Lagrangian approach can be used to determine the scaling of feeding efficacy with prey size, a key determinant of ecological niche. Specific escape strategies, such as acceleration opposite or normal to the local fluid flow, and escape response initiation soon after perception of the predator further aid prey escape. Additional experimental and theoretical studies are required to gain further insight into the these predator-prey interactions.

We note that the present study is two-dimensional. The measured flow field is a planar cross-section of a three-dimensional flow, thus the pLCS is the intersection of a two-dimensional surface with that plane. Nonetheless, the methods described here are not limited to two-dimensional flow. The Maxey-Riley equation is able to describe particle motions in three dimensions and the pLCS analysis can also apply to fully three-dimensional flow. For three-dimensional flows, the pLCS are surfaces rather than curves and they enclose a capture volume rather than an area. Tables 1 and 2 also list the size of normalized capture volume, assuming the pLCS is axisymmetric. The differences in capture volume sizes are more pronounced.

The modified Maxey-Riley equation with a forcing term can be used to study behaviours of small planktonic animals in fluid flows other than those generated by predators, such as sedimentation flows. Most previous studies on active motion of microscopic animals assume that the velocity of the animal in a flow is the vector sum of animal swimming velocity in still fluid and the ambient current velocity (e.g. Koehl \& Reidenbach 2007). The proposed equation of motion incorporates additional parameters and considers the interaction between fluid and animal on the force balance, and thus can potentially provide a more accurate way to describe the dynamics.

More importantly, the pLCS methodology proposed in the present study can be used to study transport and mixing of particles with any form of equation of motion. The method is not limited to particle motions described by the Maxey-Riley equation. It can be generalized to any other particle equation of motion, e.g. equations governing motions of reacting particles or charged particles. As long as the particle trajectories can be calculated from their equations of motion, or directly measured from experiments, pLCS can be calculated from these trajectories. This makes the method useful for a broad range of fluid mechanics applications, including multiphase and granular flows.

The authors acknowledge Cabrillo Marine Aquarium (San Pedro, CA) for providing jellyfish, and funding from the NSF Biological Oceanography Program (OCE-0623475 to J.O.D.). 


\section{REFERENCES}

Aref, H. 1984 Stirring by chaotic advection. J. Fluid Mech. 143, 1-21.

Babiano, A., Cartwright, J. H. E., Piro, O. \& Provenzale, A. 2000 Dynamics of a small neutrally buoyant sphere in a fluid and targeting in Hamiltonian systems. Phys. Rev. Lett. 84, 57645767.

Benczik, I. J., Toroczkai, Z. \& Tel, T. 2003 Advection of finite-size particles in open flows. Phys. Rev. E 67, 036303.

Costello, J. H. \& Colin, S. P. 1994 Morphology, fluid motion and predation by the scyphomedusa Aurelia Aurita. Mar. Biol. 121, 327-334.

Dabiri, J. O., Colin, S. P. \& Costello, J. H. 2006 Fast-swimming hydromedusae exploit velar kinematics to form an optimal vortex wake. J. Exp Biol. 209, 2025-2033.

FIELDS, D. M. \& YEN, J. 1997 The escape behavior of marine copepods in response to a quantifiable fluid mechanical disturbance. J. Plankton Res. 19, 1289-1304.

Franco, E., Pekarek, D. N., Peng, J. \& Dabiri, J. O. 2007 Geometry of unsteady fluid transport during fluidstructure interactions. J. Fluid Mech. 589, 125-145.

HALLER, G. 2002 Lagrangian coherent structures from approximate velocity data. Phys. Fluids 14, $1851-1861$.

Haller, G. \& Sapsis, T. 2008 Where do inertial particles go in fluid flows? Physica, D 237 573-583.

Koehl, M. A. R. \& Reidenbach, M. A. 2007 Swimming by microscopic organisms in ambient water flow. Exps Fluids 43, 755-768.

MAXEY, M. R. \& Riley, J. J. 1983 Equation of motion for a small rigid sphere in a nonuniform flow. Phys. Fluids 26, 883-889.

Michaelides, E. E. 1997 Review - The transient equation of motion for particles, bubbles, and droplets. J. Trans. ASME Fluids Engng 119, 233-247.

Rom-Kedar, V., Leonard, A. \& Wiggins, S. 1990 An analytical study of transport, mixing and chaos in an unsteady vortical flow. J. Fluid Mech. 214, 347-394.

Shadden, S. C., Dabiri, J. O. \& Marsden, J. E. 2006 Lagrangian analysis of fluid transport in empirical vortex ring flows. Phys. Fluids 18, 047105.

Shadden, S. C., Lekien, F. \& Marsden, J. E. 2005 Definition and properties of Lagrangian coherent structures from finite-time Lyapunov exponents in two-dimensional aperiodic flows. Physica D 212, 271-304.

Strickler, J. R. 1975 Swimming of planktonic Cyclops species (Copepoda, Crustacea): Pattern, movements and their control. In Swimming and Flying in Nature (ed. T. Y. T. Wu, C. J. Brokaw \& C. Brennan ), pp. 599-613 Plenum.

Sullivan, B. K., Garcia, J. R. \& Klein-MacPhee, G. 1994 Prey selection by the scyphomedusan predator Aurelia Aurita. Mar. Biol. 121, 335-341.

WigGins, S. 1992 Chaotic transport in dynamical systems. Springer. 